\title{
Literaturbericht
}

Hannes Adomeit*

\section{Moskauer Analysen zur Politik gegenüber dem Westen}

https://doi.org/10.1515/sirius-2018-2009

\section{Einleitung}

Das Moskauer Zentrum der Carnegie Stiftung hat Anfang dieses Jahres mehrere Publikationen herausgebracht, die der internationalen Öffentlichkeit gemäßigte russische Analysen der Beziehungen des Landes zum Westen präsentieren. Es geht um drei Themen: (1) die Ursachen der Zerrüttung der Beziehungen, (2) die Vermeidung einer weiteren Eskalation des Streites sowie (3) die Gefahr einer militärischen Eskalation.

\section{Ursachen der Zerrüttung des Verhältnisses zwischen Russland und dem Westen}

Andrey Kortunov ist Direktor des Russischen Rates für Internationale Beziehungen (RIAC), einer Forschungsinstitution, die von verschiedenen Ministerien, der Russischen Akademie der Wissenschaften und der Union Industrieller und Unternehmer im Jahre 2010 ins Leben gerufen wurde. Sein Artikel befasst sich mit den folgenden Fragen: Warum lagen die Sympathien des Moskauer außen- und sicherheitspolitischen Establishments im US-Wahlkampf 2016 deutlich auf der Seite Donald Trumps mit der Folge, dass seine Wahl zum 45. Präsidenten der USA mit unverhohlener Freude und sogar mit Begeisterung begrüßt wurde? Welche Gründe haben dazu geführt, dass die Erwartungen an Trump so vollständig enttäuscht wurden und die russisch-amerikanischen Beziehungen derzeit völlig zerrüttet sind? Und was könnte getan werden, um das Verhältnis zumindest auf ein erträgliches Maß zurückzuführen?

*Kontakt: Dr. Hannes Adomeit: Senior Fellow, Institut für Sicherheitspolitik an der Universität Kiel, E-Mail: hannes.adomeit@t-online.de
Für die hochgesteckten Erwartungen in Moskau macht Kortunov die folgenden Faktoren verantwortlich:

- Die russische Machtelite habe Hillary Clinton als die nachdrücklichste Befürworterin des Einsatzes von Gewalt im Nahen Osten und der Betonung der Menschenrechtskomponente in den russisch-amerikanischen Beziehungen betrachtet sowie als diejenige, die besonders deutlich Putin als Hauptgegner der westlichen Demokratien und liberaler Werte herausgestellt habe.

- Aufgrund historischer Erfahrungen hätten russische Entscheidungsträger und Experten für internationale Beziehungen angenommen, dass es leichter sei, sich mit führenden Repräsentanten der Republikanischen Partei als mit den Demokraten zu arrangieren.

- Die Wahlkampfrhetorik Trumps mit seiner wohlwollenden Einschätzung Putins und den unkonventionellen Erklärungen zu Russland, der Ukraine, Europa und der NATO hätten Anlass zu der Hoffnung auf substanzielle Veränderungen in der gesamten US-amerikanischen Außenpolitik gegeben.

- Trump sei als Befürworter einer Transaktionspolitik angesehen worden: Er würde die Beziehungen der USA zu seinen Partnern und Gegnern als normales Business behandeln, bei dem jede Partei versuche, die günstigsten Bedingungen für sich selbst herauszuschlagen.

- Trump habe immer wieder den Schutz der unmittelbaren Interessen seines Landes und dessen Souveränität betont. Dieser national-patriotische Ansatz habe sich voll und ganz im Einklang mit den Anschauungen der Moskauer Machtelite bezüglich der russischen Außenpolitik befunden.

Kortunov zeigt die „völlige Verwirrung “ auf, die jetzt unter russischen Analysten herrscht. Einige glaubten, dass der politische Neuling Trump mit seinem angesagten Kampf gegen die Staatsbürokratie und das politische Establishment Washingtons gescheitert sei. Andere seien der Meinung, er habe nie ernsthafte Anstrengungen unternommen, um die russisch-amerikanischen Beziehungen $\mathrm{zu}$ normalisieren. Seine demonstrativ „pro-russische“ 
Rhetorik sei nichts anderes als ein Trick gewesen, sich von Hillary Clinton, Barack Obama und anderen politischen Gegnern abzuheben. Und wiederum andere hielten daran fest, dass Trumps strategisches Ziel tatsächlich die Wiederherstellung der Zusammenarbeit mit Moskau sei und er nur auf die richtigen Bedingungen warte, um in diese Richtung zu gehen.

Hochinteressant ist Kortunovs Charakterisierung der spiegelbildlichen Perzeptionen in Moskau und in Washington. Konsens im russischen Establishment sei, dass die Vereinigten Staaten voll und ganz an der Zerrüttung des Verhältnisses schuld seien. Der Kreml habe dem Weißen Haus einen umfassenden Plan zur Wiederherstellung der Zusammenarbeit zwischen beiden Ländern gesandt. Der Plan habe Vorschläge zur Cybersicherheit, $\mathrm{zu}$ Handels- und Investitionsprojekten, der Beilegung von Konflikten in der Ukraine und auf der koreanischen Halbinsel sowie $\mathrm{zu}$ gemeinsamen Anstrengungen zur Bekämpfung des internationalen Terrorismus umfasst. Washington habe diese Vorschläge nicht einmal ernsthaft diskutiert. Russische Regierungsmitglieder und Diplomaten sagten infolgedessen, sie hätten „alles getan“, um das Verhältnis zu verbessern, aber ,unsere Geduld ist nun zu Ende“. Es sei jetzt besser, eine Pause einzulegen, anstatt Washington mit neuen Vorschlägen zu bombardieren.

Der Autor zumindest sieht auch Fehler auf der russischen Seite, allerdings nur „taktische” (die vermutlich ihm zufolge vorhandene, richtige „strategische“ Ausrichtung beschreibt er nicht).

- Russland habe sämtliche US-amerikanischen Vorwürfe der Einmischung in die US-Präsidentschaftswahl 2016 - unabhängig davon, ob diese tatsächlich stattgefunden hätten oder nicht - vehement zurückgewiesen. Statt zu leugnen, dass ein Problem existiere, hätte es besser sein Verständnis für die US-amerikanische Haltung demonstrieren und eine vollständige Kooperation bei der Untersuchung der Vorwürfe russischen Hackings anbieten sollen. Die Leugnung aber habe wesentlich dazu beigetragen, alle antirussischen politischen Kräfte in den Vereinigten Staaten zu mobilisieren.

- Der Kreml kommuniziere ausschließlich mit der Trump-Regierung und ignoriere ihre politischen Gegner innerhalb und außerhalb des US-Kongresses. Es wäre aber eine vernünftigere Taktik gewesen, Signale an die US-amerikanische Gesellschaft einschließlich ihrer Vertreter auf dem Capitol Hill zu senden. Zum Beispiel hätte Russland die Bereitschaft zeigen können, das Dima-Jakovlev-Gesetz über das Adoptionsverbot russischer Waisen aufzuheben beziehungsweise zu suspendieren oder einige der rus- sisch-amerikanischen Austauschprogramme, die in den letzten Jahren der Obama-Administration beendet worden seien, wiederaufzunehmen. Auch hätten USamerikanische NGOs von der Liste der „unerwünschten Organisationen“ entfernt werden können.

- Insgesamt enthielten die Vorschläge, die im März 2017 an die Trump-Regierung übermittelt wurden, keine Änderungen der Positionen zu Fragen von US-amerikanischem Interesse wie beispielsweise zur Ukraine, $\mathrm{zu}$ Syrien, Iran und der vermuteten russischen Einmischung bei den Wahlen 2016.

Die neuen Sanktionen, die Ende Juli 2017 fast einstimmig vom Repräsentantenhaus und vom Senat mit einem Gesetzentwurf verabschiedet und am 2. August von Trump trotz seines Missfallens unterzeichnet wurden, waren Kortunov zufolge „das bedeutendste Ereignis in den Beziehungen zwischen den USA und Russland im Jahr 2017“. Damit sei Russland in die gleiche Kategorie von Schurkenstaaten eingereiht worden wie Iran und Nordkorea.

In den beiden Ländern, so der Autor, habe das beiderseitige Verhältnis aufgehört, eine rein außenpolitische Angelegenheit zu sein. Es sei nunmehr, wie im Kalten Krieg, fest in der jeweiligen innenpolitischen Arena verankert. Moskau und Washington blieben auch dreißig Jahre nach dem Ende des Kalten Krieges bequeme Gegner füreinander. Ihre Konfrontation werde wieder als Instrument der politischen Mobilisierung genutzt und könne, was Russland betreffe, sogar als ein Element der nationalen Identität betrachtet werden.

Wie kommt man aus dieser fatalen Situation heraus? Kortunov setzt (für den Rezensenten überraschend) auf „die traditionelle Schlüsselrolle der strategischen Rüstungskontrolle für die bilateralen Beziehungen“. Sowohl Moskau als auch Washington hätten ernste Zweifel hinsichtlich der Zukunft des Abkommens über die Beseitigung von nuklearen Mittelstreckenwaffen und des neuen START-Vertrags. Aber wenn das Thema der strategischen Rüstungskontrolle ausgeklammert werde, könnten die russisch-amerikanischen Beziehungen einfach zerfallen. Aus diesem Grund seien die bilateralen Konsultationen zu Fragen der strategischen Stabilität, die im August 2017 in Gang gesetzt worden seien, so wichtig.

\section{Risikobegrenzungen}

Die Ansicht, dass die Rüstungskontrolle ein bedeutender Aspekt des russisch-amerikanischen Verhältnisses sei, wird auch von Dmitri Trenin, Direktor des Moskauer Car- 
negie Zentrums, in seinem Artikel geteilt, der sich mit der Frage befasst, wie man eine Eskalation im „Hybrid-Krieg“ vermeiden kann. Ihm zufolge beendete die Krise um die Ukraine ein Vierteljahrhundert der Zusammenarbeit zwischen Russland und dem Westen. Seitdem befinde sich die russische Führung in einem De-facto-Kriegszustand mit den USA. Diese Konfrontation werde oft als zweiter Kalter Krieg bezeichnet. Diese Analogie sei jedoch fehlerhaft. Die Welt habe sich seit den 1980er-Jahren zu sehr verändert, um behaupten zu können, dass der heutige Antagonismus nur eine Wiederbelebung eines alten Konflikts sei. Die neue Konfrontation sollte besser als Hybrid-Krieg (Hybrid War) beschrieben werden - ein Begriff, den Trenin wie den Kalten Krieg großgeschrieben wissen will, um seinen besonderen Platz in der Geschichte der internationalen Beziehungen hervorzuheben. Er unterscheidet ihn infolgedessen von den von Generalstabschef Valeri Gerassimov im Februar 2013 beschriebenen neuen Mitteln und Methoden der Kriegsführung des „nicht-linearen“ Kriegs, ein Begriff, der im Westen generell als „hybrider“ Krieg übersetzt wird. Trenin bezieht ihn in seiner Interpretation ganz konkret auf die russisch-amerikanischen Beziehungen.

Der Hybrid-Krieg zwischen den USA und Russland sei im Gegensatz zum Kalten Krieg zwar nicht zentral für das Weltsystem, aber sein Ergebnis werde dennoch dazu beitragen, dessen Zukunft zu gestalten. Er sei auch keineswegs das Ergebnis von Missverständnissen oder Fehleinschätzungen, sondern eher das Gegenteil, vor allem, was Russland betreffe: Die russische Führung verfolge eine Reihe von Zielen, von denen das wichtigste darin bestehe, die Rolle als Großmacht mit globaler Reichweite zu bekräftigen. In Europa solle die NATO daran gehindert werden, in das Gebiet der ehemaligen Sowjetunion, insbesondere in die Ukraine, vorzudringen. Der Kreml wolle dieses Land als Puffer zwischen Russland und der NATO verstanden wissen. Dieses vermutete Ziel ist nach Meinung des Rezensenten wohl zu kurz gegriffen. Es geht dem Kreml wohl eher um Kontrolle über die Innen- und damit auch die Außenpolitik der Ukraine. Dieser Konflikt sei kein kurzfristiges Problem, so Trenin. Es könnte eine Generation dauern, um ihn zu lösen.

Ausgelöst worden sei der Hybrid-Krieg durch die Ereignisse in der Ukraine. Die Entwicklung, die zur Absetzung des ehemaligen Präsidenten Viktor Janukovyč geführt habe, sei von Russland als Bedrohung wahrgenommen worden - als Versuch der USA, den für Russland wichtigsten strategischen Puffer zu beseitigen, die von Moskau angestrengte Integration im postsowjetischen Eurasien $\mathrm{zu}$ verhindern und eine Barriere zum Rest von Europa $\mathrm{zu}$ errichten. Für die USA überraschend reagierte Putin mit der Anwendung von Gewalt, zuerst, um die Krim für Russ- land zu sichern und dann, um eine Rebellenhochburg im Donbass aufzubauen.

Was sind nun laut Trenin die Merkmale des russischamerikanischen Hybrid-Kriegs? Seine Besonderheit sei, dass er in einer wahrhaft globalen, im wahrsten Sinne des Wortes grenzenlosen Umgebung ausgefochten werde. Der Konflikt sei nicht länger durch Mauern oder andere staatlich auferlegte Barrieren eingeschränkt. Traditionelle Unterscheidungen zwischen Strategie und Taktik seien hinfällig. Die Art und Anzahl der Akteure sei weiter gefasst als im Kalten Krieg, sie reichten von nationalen Regierungen und transnationalen Konzernen über nichtstaatliche Akteure bis hin zu Privatpersonen. Der Krieg werde gleichzeitig in verschiedenen Bereichen, auf verschiedenen Ebenen und im nie endenden 24-StundenNachrichtenzyklus ausgetragen. Dieser Aspekt der Kriegsführung gelte insbesondere für das Informationsfeld, das sich von Cyber-Konflikten und dem Einsatz künstlicher Intelligenz bis hin zum Einsatz von Propaganda und Fake News erstrecke.

Weiter beinhalte der Hybrid-Krieg Maßnahmen, politische Veränderungen in anderen Ländern zu bewirken. Dazu gehörten die organisatorische und finanzielle Förderung bestimmter Parteien oder Volksbewegungen, Demonstrationen und Straßenproteste, die Einmischung in Wahlen, Bemühungen, sensible Informationen $\mathrm{zu}$ hacken sowie kompromittierende oder beschädigende Materialien und gefälschte Nachrichten zu verbreiten, die Begünstigung von Rufmorden und der Erlass personenbezogener Sanktionen wie Visabeschränkungen, Beschlagnahme von Vermögenswerten und Inhaftierung oder Abschiebung politischer Gegner.

Militärische Macht sei auch im Hybrid-Krieg wichtig. Allerdings seien Mittel und Möglichkeiten ihrer Anwendung anders als im Kalten Krieg, die Bedeutung millionenstarker Armeen sei zurückgegangen. Die nukleare Abschreckung zwischen Russland und dem Westen bleibe bestehen, aber auf einem niedrigeren und stabileren Niveau. Die heutigen Risiken der Fehleinschätzung beruhten auf potenziellen Zwischenfällen mit konventionellen Streitkräften. An der Grenze zu den NATO-Staaten habe sich eine militärische Pattsituation gebildet, aber diese habe keine Ähnlichkeit mit den Kräften, die sich während des Kalten Kriegs gegenüberstanden. Der Schwerpunkt des Konflikts liege auf der Entwicklung neuer militärischer Technologien und Kriegsführung im Weltraum und im Cyberspace.

Ein letztes wichtiges Merkmal des Hybrid-Krieges im Hinblick auf die russisch-amerikanische Konfrontation sei die Asymmetrie der Fähigkeiten der beiden Kontrahenten. In den 1970er-Jahren sei die Sowjetunion den Verei- 
nigten Staaten in Bezug auf nukleare und konventionelle militärische Macht gleichgestellt gewesen. Über seine eigene riesige Landmasse und unmittelbare Einflusssphäre in Osteuropa hinaus habe es in vielen westlichen Ländern und in der Dritten Welt über eine beträchtliche ideologische Macht und ein Bündnissystem in Afrika, Asien, Europa, Lateinamerika und dem Nahen Osten verfügt. Im Gegensatz dazu habe das heutige Russland nur wenige formelle Verbündete, keine Satellitenstaaten und eine Handvoll Protektorate, wenn man die selbsternannten Staaten Abchasien, Donbass, Südossetien und Transnistrien mit einbeziehe. Es habe keine Ideologie, die man mit dem umfassenden Dogma des MarxismusLeninismus vergleichen könnte, und obwohl es immer noch eine nukleare Supermacht sei, bleibe es bei den nicht-nuklearen militärischen Fähigkeiten weit hinter den Vereinigten Staaten zurück. Wirtschaftlich betrachtet sei Russland mit geschätzten 1,5 Prozent des globalen Bruttoinlandsprodukts ein Zwerg.

Um zu bestehen, müsse sich Russland auf seine wenigen komparativen Vorteile stützen und versuchen, diese optimal zu nutzen. $\mathrm{Zu}$ diesen Vorteilen gehöre (1) die geografische Nähe zu einigen der wichtigsten Schauplätzen, wie die Krim und die Ost-Ukraine, wo Russland auch über Eskalationsdominanz verfüge, (2) das russische politische System, das geheimes, schnelles und entschlossenes Handeln ermögliche, und (3) die Bereitschaft, höhere Risiken vor dem Hintergrund einer Nationalkultur einzugehen, die in der Vergangenheit höhere Verluste in der Verteidigung oder zum Schutz des Mutterlandes geduldet habe.

Im Schlussteil des Artikels befasst sich Trenin mit der Frage, wie man vermeiden könne, dass der Hybrid-Krieg zu einem hybriden Krieg (im Sinne Gerassimovs) oder zu einem konventionellen Krieg eskaliert. Die Wege, die er sieht, sind Vorbeugung von Vorfällen, Vertrauensbildung und Rüstungskontrolle.

- Relativ am leichtesten, soweit der Wille vorhanden sei, könnten risikobehaftetes Verhalten vermieden und Vorfällen vorgebeugt werden. Um eine Eskalation zu vermeiden, sollten die Militärbehörden Russlands und der NATO über zuverlässige Kommunikationskanäle verfügen. Der ursprünglich als Vehikel der Zusammenarbeit konzipierte NATO-Russland-Rat sollte für diese neue Rolle angepasst werden. Russische und NATOKommandeure in Operationsgebieten zu Land, auf See und in der Luft müssten ebenfalls über Notfallmittel verfügen. Auf höchster militärischer Ebene sollte der russische Chef des Generalstabs eine direkte Verbindung zum Vorsitzenden der Vereinigten Stabschefs der USA haben. Regelmäßige persönliche Treffen dieser
Spitzenbeamten, die bereits dreimal im Jahr 2017 abgehalten worden seien, seien ebenfalls wichtig, um eine professionelle Beziehung aufzubauen.

- Die Vertrauensbildung umfasse eine Reihe von Maßnahmen, die von der NATO und den WarschauerPakt-Staaten am Ende des Kalten Kriegs vereinbart und im regelmäßig überarbeiteten Wiener Dokument kodifiziert worden seien. Dazu zählten Meldungen vor größeren militärischen Übungen, die Einladung von Beobachtern zu diesen Aktivitäten und Beratungen in Bezug auf Luftüberflüge von Gebieten der teilnehmenden Parteien. Diese Maßnahmen seien zwar noch vorhanden, würden aber widerwillig und mit einigen Ausnahmen angewendet. Russland, das seine Fähigkeiten wiedererlangen wolle, habe sowohl kurzfristig anberaumte als auch längerfristig geplante Militärmanöver durchgeführt, deren Parameter knapp unter der Größen- und Umfangschwelle liegen, die eine verbindliche Voranmeldung oder die Einladung von Beobachtern erfordern würde. In der neuen asymmetrischen Situation habe Russland Grund zu der Annahme, dass es von Vorteil sein könne, wenn einige seiner Aktionen mehrdeutig oder unvorhersehbar seien.

- Im Kalten Krieg sei die Rüstungskontrolle eines der wichtigsten Instrumente des Konfrontationsmanagements gewesen. Dies sei im Hybrid-Krieg nicht mehr der Fall. Rohe militärische Macht spiele eine weitaus geringere Rolle als früher. Heute existiere ein neues geopolitisches Umfeld. Im militärtechnologischen Bereich hätten mobile Langstreckenwaffensysteme mit ihrer stark verbesserten Präzision quantitative und regionale Beschränkungen nahezu bedeutungslos gemacht. Auch seien die Fähigkeiten der Waffensysteme wichtiger geworden als ihre Anzahl, wodurch traditionelle Paradigmen der Rüstungskontrolle aufgehoben worden seien. Umgekehrt ist allerdings für Trenin klar, dass die Aufhebung bestehender Rüstungskontrollvereinbarungen zu einer Verschärfung der russisch-amerikanischen Konfrontation führen würde. Das würde eintreten, wenn beispielsweise das 1987 geschlossene Abkommen über das Verbot von Nuklearwaffen mittlerer Reichweite (INF-Vertrag) außer Kraft gesetzt würde (was de facto passiert sein könnte, da laut US-amerikanischen Erkenntnissen Russland einen bodengestützten Marschflugkörper entsprechender Reichweite entwickelt hat). Ähnlich verhielte es sich mit dem neuen START-Vertrag für strategische Rüstungsreduzierungen aus dem Jahre 2011, der 2021 auslaufe. Würde er nicht erneuert, bedeute dies eine endgültige Entkopplung zwischen Moskau und Washington im nuklearstrategischen 
Bereich, was eine neue Ära ungeregelter Verhältnisse bei der Rüstungskonkurrenz einleiten könnte. Um eine weitere schwerwiegende Verschlechterung der Beziehungen zu vermeiden, müsste also der INF-Vertrag beibehalten und, was den neuen START-Vertrag betreffe, zumindest seine Geltungsdauer verlängert werden.

Trenin ist allerdings realistischer als Kortunov, was Rüstungskontrolle tatsächlich leisten könnte, um die russischamerikanische Konfrontation zu entschärfen. Russland befindet sich derzeit in einer andauernden Phase umfassender Modernisierung seiner Waffensysteme, vor allem seiner nuklearstrategischen Waffen. Putin hat darauf mit Schaubildern am 1. März 2018 in seiner Rede zur Lage der Nation eindrucksvoll hingewiesen und dies mit dem Test einer Sarmat-Interkontinentalrakete am 30. März bekräftigt. Desgleichen hat Trump die Modernisierung des US-amerikanischen nuklearstrategischen Potenzials zu einer Priorität seiner Amtszeit erklärt. Davon abgesehen wären vermutlich in absehbarer Zukunft Aushandlung, Abschluss und endgültige Ratifizierung eines neuen Atomwaffenabkommens zwischen den USA und Russland politisch unmöglich.

Ein anderer Artikel Trenins ist Teil eines Projekts mit dem Titel „Begrenzung des Risikos einer Ost-West-Kollision: Praktische Ideen zur europäischen Sicherheit“, das vom britischen Außenministerium gefördert wird.

Was sind die „praktischen Ideen“, die Trenin entwickelt, um aus der gegenwärtigen tiefen Krise in den Beziehungen zwischen Russland und dem Westen herauszukommen? Sein Lösungsansatz ist die Herstellung eines „Neuen Equilibriums“. Er geht davon aus, dass sich die „Neuheit des hybriden Krieges abschleifen und die Emotionen, die seinen Ausbruch umgeben haben, nachlassen werden“. (In diesem Artikel bezieht sich Trenin auf den „nicht-linearen“ Krieg à la Gerasimov, also den „hybriden“ Krieg in Kleinbuchstaben.) Dies würde die Voraussetzungen dafür schaffen, dass sich die Führungspersönlichkeiten Russlands und des Westens gelassener mit den Quellen, Ergebnissen und Kosten ihrer Konfrontation befassen könnten. Ein erster Schritt müsste darin bestehen, dass man damit aufhöre, ,an die eigene Propaganda zu glauben, egal ob sie staatlich verordnet oder von Mainstream-Media betrieben wird“. Dazu würde auch gehören, dass man die schlimmsten Praktiken der anderen nicht imitiere, „etwa das Streben nach einem Informationsmonopol oder Organisationen und Personen als ,ausländische Agenten` zu brandmarken.“

In einem zweiten Schritt müssten sich Russland und der Westen bemühen, dort zu kooperieren, wo ihre Inter- essen dies rechtfertigten, und damit eine Gegenbewegung einleiten, die ihren Konflikt abschwächen könnte. Geografisch existierten solche Bereiche potenzieller Kooperation in Europa, an der Peripherie wie beispielsweise in Berg-Karabach, vom Nahen Osten über Nordafrika bis zur Arktis - und auch ,anderswo auf der Welt“. Funktional könnte sich die Zusammenarbeit auf die Nichtverbreitung von Kernwaffen, Terrorismusbekämpfung und Cybersicherheit richten.

Trenin kommt dann zum Kern des Equilibriums, den er in der folgenden Formel zusammenfasst: „Keine weitere NATO-Erweiterung in den ehemaligen sowjetischen Raum und keine Beschränkung der Annäherung der ehemaligen Sowjetrepubliken an die Europäische Union." Ein derartiger Kompromiss sei ohnehin für praktisch alle ernsthaften Beobachter offensichtlich. Die NATO habe keinen Grund, weiter $\mathrm{zu}$ expandieren, wohlwissend, dass dies nur $\mathrm{zu}$ einem viel schlimmeren Konflikt führen würde als 2014. Und Russland müsste entscheiden, sich einer Annäherung nicht nur der Ukraine, Georgiens und der Republik Moldau, sondern auch von Belarus und Armenien an die Europäische Union nicht in den Weg zu stellen. Die Mitglieder der Östlichen Partnerschaft der EU, die auch Mitglieder der Eurasischen Wirtschaftsunion (EEU) seien, sollte Moskau als Brücke zwischen der EEU und der EU betrachten.

Nach Auffassung des Rezensenten ist Trenins Lösungsansatz zwar weniger kritikwürdig als der von der Hessischen Stiftung für Friedens- und Konfliktforschung (HSFK) vorgeschlagene „plurale Frieden“ mit der De-factoAnerkennung einer russischen Einflusssphäre in Europa, aber sehr viel realistischer ist er auch nicht. Eine weitere Runde der NATO-Osterweiterung ist wegen der ungelösten Konflikte in der Ukraine (Krim, Donezk, Luhansk) und Georgien (Abchasien, Südossetien) ohnehin bereits ausgeschlossen. Und mit Blick auf Russland ist der implizit in dem Vorschlag enthaltenen These zuzustimmen, dass ein neues Gleichgewicht möglich wäre, wenn sich die russische Politik im postsowjetischen Raum grundlegend ändern würde. Dafür gibt es allerdings bisher keine Anhaltspunkte. Russland zeigt derzeit keine Bereitschaft, seinen Nachbarstaaten eine europäische Perspektive zu eröffnen - warum sollte es auch, zumal es sich selbst diesen Weg verbaut?

\section{Gefahr einer militärischen Eskalation}

Aleksandr Khramchikhin ist stellvertretender Direktor des Instituts für politische und militärische Analysen (IPMA) 
in Moskau, das 1996 gegründet wurde und sich als unabhängige, nichtstaatliche Forschungsorganisation bezeichnet. Es arbeitet auch mit staatlichen Stellen zusammen und liefert für sie Expertisen, die nicht unbedingt auf Regierungslinie liegen.

Der Kern seiner Argumentation ist wie folgt: Der weit verbreitete Diskurs über eine angeblich höchstgefährliche militärische Situation, die kurz davor stehe, außer Kontrolle zu geraten, habe wenig oder überhaupt nichts mit der Wirklichkeit zu tun. Beide Seiten übertrieben die Eskalationsrisiken der militärischen Bewegungen. Das militärische Gleichgewicht zwischen Russland und der NATO sei stabil, die Gefahr der Eskalation nähere sich kaum einem kritischen Niveau und militärisch müsse wenig getan werden, um Risiken zu entschärfen. Ein militärischer Konflikt in Europa sei völlig unrealistisch. Keine Seite habe Kampferfahrung oder ausreichende offensive Fähigkeiten und zeige auch keine Bereitschaft, solche Fähigkeiten zu erwerben und sie für Offensivoperationen einzusetzen. Eine militärische Konfrontation zwischen Russland und der NATO könne nur in einem Szenario auftreten: Wenn die Streitkräfte Russlands und der USA in Syrien zusammenstießen und der Konflikt dann unkontrolliert eskalieren würde. Khramchikhins Diagnose unterscheidet sich radikal von dem Narrativ des Kremls und des russischen Generalstabs über das „Vordringen von NATO-Stoßkräften und Infrastruktur immer näher an unsere Grenzen“ und eine ernst zu nehmende Sicherheitsbedrohung des Landes durch die NATO, aber auch von der Trenins, der, wie oben ausgeführt, von einem „Kriegszustand“ in den russischamerikanischen Beziehungen spricht.

Aufschlussreich sind die Tabellen des Autors, in denen er die militärischen Fähigkeiten an Waffen und Personal im Vergleich von NATO zu Russland als auch zur Zeit des Kalten Kriegs (NATO und Warschauer Pakt) zusammenstellt und interpretiert.

Hinsichtlich der NATO hält er es für bemerkenswert, dass sich die Anzahl ihrer Mitgliedstaaten seit dem Ende des Kalten Krieges zwar fast verdoppelt habe, Kampfkraft und Einsatzfähigkeiten des Bündnisses aber im Vergleich zu den 1980er-Jahren, als die Ost-West-Spannungen hoch gewesen seien, bedeutsam verringert hätten. Zwar seien neue Waffen entwickelt und eingeführt sowie Fortschritte in den militärischen Führungsfähigkeiten gemacht worden, aber die Arsenale der meisten europäischen Länder wiesen nur minimale qualitative Verbesserungen auf, welche die Reduzierungen ihrer militärischen Fähigkeiten nicht kompensieren könnten. Größere Anschaffungen von militärischer Ausrüstung beschränkten sich hauptsächlich auf gepanzerte Schützenpanzerwagen (APCs), die in Expeditionskriegen eingesetzt werden sollen.
Die US-Militärpräsenz in Europa seit dem Ende des Kalten Krieges sei ebenfalls enorm zurückgefahren worden. So habe das US-Militär zu Beginn des Jahres 2016 in Deutschland zehn Brigaden stationiert, aber nur zwei davon (das 2. Kavallerieregiment und die 12. Kampffliegerbrigade) könnten als kampftauglich eingestuft werden; die restlichen acht seien reine Unterstützungseinheiten. Die US-Luftwaffe habe im Jahr 2017 in Deutschland, Italien und Großbritannien über neun Regimenter (wings, vergleichbar bei den Landstreitkräften mit einer Brigade) verfügt. Diese seien aber in erster Linie wiederum nur Unterstützungseinheiten, in denen es nur insgesamt sechs Kampfgeschwader (combat squadrons) gebe.

In letzter Zeit habe die NATO einige Streitkräfte nach Osteuropa verlegt, zum Teil auf Rotationsbasis. Die Stationierungen seien lediglich symbolischer Art, wofür es mehrere Gründe gebe. Erstens hätten nicht einmal die Vereinigten Staaten, geschweige denn ihre europäischen Verbündeten, ausreichende militärische und finanzielle Ressourcen, um große militärische Einheiten in Osteuropa zu stationieren. Zweitens seien die Regierungen der NATOLänder trotz ihrer harten antirussischen Rhetorik über die Aussicht auf eskalierende Spannungen mit Moskau besorgt, und eine solche Eskalation wäre wahrscheinlich, wenn das Bündnis in den Ländern entlang Russlands Grenzen bedeutende Streitkräfte stationieren würde.

Immerhin sei im Rahmen von NATO-Übungen eine U.S.-Stryker-Brigade in mehreren osteuropäischen Ländern herumkutschiert. ${ }^{1}$ Die Brigade, die mit eher dünnwandigen Schützenpanzerwagen (APCs) ausgerüstet sei, könne man allerdings als Machtdemonstration nicht ernst nehmen. Stryker-Brigaden könnten vielleicht sinnvoll gegen schlecht ausgestattete, unterentwickelte Armeen eingesetzt werden, aber nicht gegen gut organisierte und trainierte Kampfeinheiten. Es sei „grotesk“, wenn Repräsentanten der NATO solche begrenzten Übungen mit Erklärungen untermalten, dass man damit eine klare und starke Botschaft an Russland sende.

Die Kürzungen der militärischen Hardware und der materiellen Schwächung der Kampfkraft insbesondere der europäischen NATO-Mitglieder müssten in den Zusammenhang mit dem Schwinden von Kampfmoral und Kampfbereitschaft gestellt werden. Die gesellschaftliche Verfassung des Westens, in größerem Maße in Europa als in den Vereinigten Staaten, sei von „Hedonismus, Pazifismus, Postmodernismus, [übertriebener] Toleranz und politischer Korrektheit" gekennzeichnet. Zur Untermauerung dieser These zitiert Khramchikhin eine Umfrage des

1 Ein Stryker Brigade Combat Team, abgekürzt SBCT, ist eine verstärkte Brigade der U.S. Army mit ca. 3.900 Soldaten. 
Pew-Forschungszentrums aus dem Jahre 2016, der zufolge die Europäer, mit Ausnahme der Polen und der Holländer, gegen Erhöhungen ihrer Verteidigungsausgaben seien. Im darauffolgenden Jahr habe eine weitere Pew-Umfrage ergeben, dass die Bereitschaft der Europäer, im Ernstfall einem NATO-Verbündeten gegen Russland zu Hilfe zu kommen, gering sei, wobei die geringste Neigung in Deutschland, Großbritannien und Spanien festzustellen gewesen sei. Derartige Haltungen sowie schrumpfende NATO-Streitkräfte und Arsenale bedeuteten, dass erhebliche Verluste an Leben oder Ausrüstung unannehmbar seien. Der Verlust von nur wenigen Panzern und Flugzeugen würde schon als Katastrophe betrachtet, vergleichbar einem früheren Verlust eines Schlachtschiffs oder einer Panzerdivision.

Die russische Führung selbst habe eine skeptische Sicht auf die NATO, die sich radikal von ihrer offiziellen Propaganda unterscheide: Glaubten sie ihrer eigenen Propaganda, hätten sie in Südossetien, Georgien, auf der Krim, in der Ukraine und in Syrien niemals Gewalt angewandt.

Wie bewertet Khramchikhin die Entwicklungen in Russland? Er stellt fest, dass die jüngste militärische Modernisierung des Landes auf einer sehr niedrigen Basis begonnen habe, da an die russischen Streitkräfte etwa fünfzehn Jahre - von Mitte der 1990er- bis fast Ende der 2000er-Jahre - fast keine Waffen außer ICBMs und SLBMs überstellt worden seien. Ende der 2000er-Jahre habe sich das russische Militär einem völligen Zusammenbruch genähert. Die russische Armee habe kurz davorgestanden, sich in Gruppen von mit Kalaschnikows bewaffneten Männern aufzulösen. Angesichts dieser Aussicht habe die russische Regierung keine andere Wahl gehabt, als ein breites Programm zur Beschaffung von Waffen und Ausrüstung zu starten. Infolgedessen habe auch das Tempo russischer militärischer Hardware-Upgrades in den vergangenen Jahren das der NATO überholt.

Der Autor geht auch auf die westlichen Vorstellungen ein, dass Russland einen Krieg, wenn auch nur einen „hybriden“, gegen die baltischen Staaten oder Polen führen könnte. Er hält die bloße Idee einer russischen Aggression gegen ein NATO-Land für ,absurd“ und nicht lohnend, sie „auch nur hypothetisch“ zu diskutieren. Alle diese Szenarien gehörten zur Kategorie von Propaganda oder Wahnvorstellungen und beruhten auf einem völligen Unverständnis dessen, was im postsowjetischen Raum in den letzten dreißig Jahren geschehen sei.

Das betreffe auch die 2017 im Westen aufgeflammte Diskussion, ob das jüngste „Zapad“-Militärmanöver nicht fließend zu einer tatsächlichen Invasion der baltischen Staaten oder Polens hätte führen können. Dies zeige nur, wie stark sich die Grenze zwischen Analyse und Propaganda oder Paranoia innerhalb der NATO verwischt habe. Das Konzept des „hybriden Krieges“ gehöre ebenfalls zur Kategorie der Propaganda. Es gebe keine wissenschaftliche Definition des hybriden Krieges, und jeder Versuch, ihn zu definieren, führe zu dem Schluss, dass auch der hybride Krieg ein Krieg sei. Dieser sei immer eine Kombination von regulären und irregulären bewaffneten Feindseligkeiten sowie von Wirtschafts- und Informationskrieg gewesen, auch wenn seine Mischung sich von Konflikt zu Konflikt unterschieden habe.

Wie unsinnig westliche Vorstellungen von russischen Invasionsvorbereitungen seien, lasse sich auch an der Verteilung von Streitkräften und Waffen in Russland nachweisen. Schon vor der Ukraine-Krise habe der südliche Militärdistrikt, der direkt an die Ukraine und das Schwarze Meer angrenze, gegenüber dem westlichen Militärdistrikt, der Großstädte wie Moskau und St. Petersburg einschließe, Vorrang gehabt. Diese Priorität habe sich auch nach der Ukraine-Krise nicht geändert.

Die einzige neue russische Militäreinheit, die in den Grenzgebieten zu den NATO-Staaten gebildet worden sei, sei die 15. Armee-Luftwaffenbrigade in der Stadt Ostrov (Region Pskov). In Alakurtti (Region Murmansk) sei eine weitere militärische Einheit, die 80. motorisierte Schützenbrigade, gegründet worden. Dabei handle es sich aber um eine rein experimentelle Einheit, deren Aufgabe die Entwicklung von Kampfmethoden in der Arktis sei. Weder in Leningrad noch in Kaliningrad seien neue militärische Einheiten gebildet worden und die Modernisierung bestehender Einheiten an diesen Orten schreite ohne Eile voran. Dies treffe auch auf die Ersetzung der veralteten Toschka-U mit der Iskander-Rakete zu. Von den elf mit Iskander ausgerüsteten Raketenbrigaden stünden vier vor Russlands „strategischem Partner“ China. Nur drei Iskander-Brigaden seien vor dem ,aggressiven Block“ der NATO stationiert, eine davon in Šuja in der Region Ivanovo, weit von den Kontaktlinien zur NATO entfernt.

Diese Situation stehe in krassem Gegensatz zu den Entwicklungen entlang der russisch-ukrainischen Grenze, von der Stadt Briansk bis nach Rostov am Don. Seit 2016 seien drei neue motorisierte Schützendivisionen (3., 144. und 150.) gebildet worden. Außerdem sei die 53. Flugabwehrraketenbrigade (die erste solche Brigade der russischen Streitkräfte, die mit Buk-M3-Flugabwehrraketensystemen ausgerüstet ist) gegründet und in die Strukturen der 20. Armee des Westlichen Militärbezirks und 8. Armee des Südlichen Militärbezirks eingefügt worden.

Wie Kortunov und Trenin beschäftigt sich auch Khramchikhin mit der Frage, wie die gegenwärtige Krise in den Beziehungen zwischen Russland und dem Westen 
$\mathrm{zu}$ lösen wäre. Seine Ideen sind dieselben wie die seiner beiden Forscherkollegen.

- Die NATO sollte sich formell verpflichten, die Mitgliedschaft in der Allianz für keine weitere der ehemaligen Sowjetrepubliken anzustreben.

- Der Westen sollte eine „realistische Perspektive“ auf das unerledigte Geschäft nicht-anerkannter Territorien entwickeln und die Situation auf der Krim, in Abchasien und in Südossetien als vollendete Tatsache anerkennen. Entsprechende rechtliche Bestimmungen müssten durch Resolutionen des UN-Sicherheitsrates - erforderlichenfalls mittels neuer Referenden - geschaffen werden.

- Der Donbass und Transnistrien sollten in der Ukraine beziehungsweise in der Republik Moldau einen Sonderstatus erhalten.

- Die anti-russischen Sanktionen sollten aufgehoben werden, da sie wenig Einfluss auf Russland hätten, aber jegliche Aussicht auf einen Dialog unter Gleichen ausschlössen.

- Die Vereinigten Staaten sollten aufhören, sich in Russlands innere Angelegenheiten einzumischen und die pro-westliche demokratische Opposition zu unterstützen, denn dadurch könne man diese als „ausländische Agenten“ diskreditieren.

\section{Ausblick}

Die Artikel wurden vor dem Giftgasangriff auf Sergej Skripal und seine Tochter Julia in Salisbury verfasst, es ist aber nicht zu erkennen, dass die drei Autoren vor diesem Ereignis erwarteten, dass sich in naher Zukunft irgendetwas an dem desolaten Zustand der Beziehungen zwischen Russland und dem Westen ändern könnte. Allesamt machen sie zwar den Vorschlag, dass die NATO auf eine weitere Osterweiterung verzichten sollte, zumindest Kortunov und Trenin halten aber eine formelle Zusage der westlichen Allianz für ausgeschlossen. Ihre Forderung an den Kreml, er möge Bereitschaft zeigen, seinen Nachbarstaaten Freiraum zu gewähren und ihnen eine europäische Perspektive zu gestatten, muss ebenfalls als unrealistisch erscheinen. Schließlich entstand der scharfe Konflikt in und um die Ukraine im Jahre 2013 nicht durch NATO-Osterweiterungspläne, sondern infolge des russischen Widerstands gegen den Abschluss eines ,tiefen und umfassenden“ Assoziierungsabkommens der Ukraine mit der EU. Dieser Konflikt ist Folge einer von Putin nach den Präsidentschaftswahlen im März 2012 getroffenen Grundsatzentscheidung, von der von Dmitri Medvedev in seiner Eigenschaft als Präsident propagierten sozio-ökonomischen Modernisierung zur national-patriotischen Mobilisierung überzugehen.

Die Autoren sind dem Rezensenten persönlich bekannt und sind eher prowestlich und Putin-kritisch eingestellt beziehungsweise waren es früher. Es ist wohl ein Merkmal der autoritären Entwicklung in Russland, dass sie nicht auf diese und andere innenpolitische Faktoren als Voraussetzung für eine grundlegende Veränderung der Beziehungen zwischen Russland und dem Westen eingehen.

\section{Besprochene Literatur}

Andrey Kortunov: Disillusionment and Missed Opportunities: Russian-U.S. Relations in 2017, Carnegie Endowment for International Peace, Washington, D.C., Februar 2018 http://carnegieendowment.org/2018/02/23/disillusionmentand-missed-opportunities-russian-u.s.-relations-in-2017-pub75626

Dmitri Trenin: Avoiding U.S.-Russia Military Escalation During the Hybrid War, Carnegie Moscow Center, Januar 2018 http:// carnegie.ru/2018/01/25/avoiding-u.s.-russia-militaryescalation-during-hybrid-war-pub-75277

Dmitri Trenin: European Security: From Managing Adversity to a New Equilibrium, Carnegie Moscow Center, Februar 2018 http://carnegie.ru/2018/02/22/european-security-frommanaging-adversity-to-new-equilibrium-pub-75606

Aleksandr Khramchikhin: Rethinking the Danger of Escalation: The NATO-Russia Military Balance, Carnegie Endowment for International Peace, Washington, D.C., Januar 2018 http:// carnegieendowment.org/2018/01/25/rethinking-danger-ofescalation-russia-nato-military-balance-pub-75346 